\title{
CENTRAL LIMIT THEOREM FOR SIGNED DISTRIBUTIONS ${ }^{1}$
}

\author{
KENNETH J. HOCHBERG
}

\begin{abstract}
This paper contains an improved version of existing generalized central limit theorems for convergence of normalized sums of independent random variables distributed by a signed measure. It is shown that under reasonable conditions, the normalized sums converge in distribution to "higher-order" analogues of the standard normal random variable, in the sense that the density of the limiting signed distribution is the fundamental solution of a higher-order parabolic partial differential equation that is a generalization of the heat equation.
\end{abstract}

1. Introduction. Probability theory is of ten thought of as the study of nonnegative bounded measures. However, several theorems usually considered to be purely probabilistic are, in fact, true in more general contexts. Specifically, several authors have extended such results to signed measures. For example, Hochberg [4] derives generalizations of the Brownian motion or Wiener process to higher-order signed stochastic processes, an analogue of the Itô stochastic calculus for these signed processes, and a probabilistic-style analysis of the spectral properties of higherorder elliptic operators.

Similarly, the central limit theorem for convergence of normalized sums of random variables to the Gaussian distribution has been extended to the case where the random functions are distributed by a signed measure. Zukov [8] derives such a theorem for difference operators in a study of a problem in numerical analysis. Studnev [6] states, without proof, other generalizations, with an application to probability theory itself. Hersh [3] gives a more complete study of generalized central limit theorems, though his results do not apply to the cases $n=0(\bmod 2)$ in what follows; that is, Hersh's theorems exclude the cases where the signed distribution has a density function which is the fundamental solution of the parabolic partial differential equation (2) below whose order is a multiple of four. Our techniques include these cases.

In an earlier paper (Hochberg [4]), we derived two such central limit theorems, which we restate in the following two propositions. Here

$$
\begin{aligned}
p_{2 n}(t, x) & =(2 \pi)^{-1} \int_{-\infty}^{\infty} e^{i x \xi} \exp \left\{-\xi^{2 n} t\right\} d \xi \\
& =\left(\exp \left\{-\xi^{2 n} t\right\}\right)^{\wedge},
\end{aligned}
$$

where $\hat{f}(\xi)=(2 \pi)^{-1} \int_{-\infty}^{\infty} e^{i x \xi} f(x) d x$ denotes the Fourier transform of $f$, is the fundamental solution of the even-order parabolic partial differential equation

Received by the editors August 8, 1979.

AMS (MOS) subject classifications (1970). Primary 60F05; Secondary 60G20.

Key words and phrases. Central limit theorem.

${ }^{1}$ Research supported in part by National Science Foundation Grant MCS 78-02144. 


$$
\frac{\partial u}{\partial t}=(-1)^{n+1} \frac{\partial^{2 n} u}{\partial x^{2 n}}, \quad n>2
$$

As such, it is a higher-order analogue of the Gaussian, or normal, probability density function

$$
(2 \pi t)^{-1 / 2} \exp \left\{-x^{2} / 2 t\right\}=\left(\exp \left\{-\frac{1}{2} \xi^{2} t\right\}\right)
$$

which is itself the fundamental solution of the heat equation $\partial u / \partial t=\frac{1}{2} \partial^{2} u / \partial x^{2}$.

We note here for future reference that $p_{2 n}(t, x), n>2$, is a signed function, and that

$$
\begin{aligned}
\text { (i) } \int_{-\infty}^{\infty} p_{2 n}(t, x) d x & =1, \\
\text { (ii) } \int_{-\infty}^{\infty} x^{j} p_{2 n}(t, x) d x & =0 \text { for } j=1,2, \ldots, 2 n-1, \\
\text { (iii) } \int_{-\infty}^{\infty} x^{2 n} p_{2 n}(t, x) d x & =(-1)^{n+1}(2 n) ! t
\end{aligned}
$$

Notation. By $C_{\downarrow}^{\infty}$ we denote the Schwarz class of infinitely differentiable functions $f$ with $\lim _{|x| \rightarrow \infty} x^{k}\left(d^{j} / d x^{j}\right) f(x)=0$ for each $k, j>0$.

Proposition 1.1 (Theorem 5.1 of Hochberg [4]). Let $x_{i}, i=1,2, \ldots$, be independent random variables, identically distributed by a signed measure $\mu$, such that

$$
\begin{aligned}
\int_{-\infty}^{\infty} d \mu & =1, \\
\int_{-\infty}^{\infty} x_{i}^{j} d \mu & =0, \quad j=1,2, \ldots, 2 n-1, \\
& =(-1)^{n+1}(2 n) !, \quad j=2 n, \\
\int_{-\infty}^{\infty}\left|x_{i}\right|^{2 n+1}|d \mu| & <\infty .
\end{aligned}
$$

Let $S_{n}=x_{1}+x_{2}+\cdots+x_{n}$. Then, for any function $\varphi \in C_{\downarrow}^{\infty}$ whose Fourier transform $\hat{\varphi}$ has compact support, we have

$$
\lim _{k \rightarrow \infty} E\left\{\varphi\left(\frac{S_{k}}{k^{1 / 2 n}}\right)\right\}=\int_{-\infty}^{\infty} \varphi(x) p_{2 n}(1, x) d x
$$

Proposition 1.2 (Theorem 5.2 of Hochberg [4]). Let $x_{i}(i=1,2, \ldots), S_{n}$, and $\mu$ be as in Proposition 1.1, and let the signed measure $\mu$ have a density $f(x)$ with Fourier transform $\hat{f}$ such that $|\hat{f}|<(2 \pi)^{-1}$. Then, for any $\varphi \in C_{\downarrow}^{\infty}$, we have

$$
\lim _{k \rightarrow \infty} E\left\{\varphi\left(\frac{S_{k}}{k^{1 / 2 n}}\right)\right\}=\int_{-\infty}^{\infty} \varphi(x) p_{2 n}(1, x) d x .
$$

These theorems are proven using Fourier-analytic techniques; dependence upon that approach is reflected in the statement of the results. For an application of Proposition 1.2 to the theory of random evolutions and related semi-Markov processes, see Kertz [5].

The signed function $p_{2 n}(1, x)$ can be taken to be the density of a related signed distribution function $\boldsymbol{P}_{2 n}$. For a complete discussion of such signed distributions, 
see Hochberg [4]. When viewed in this context, we will say that the random variable $\delta_{2 n}$ distributed by $P_{2 n}$ is the higher-order analogue of the "standard normal" random variable $\Re(0,1)$.

In this paper, we use a different method to prove a new version of the central limit theorem for signed distributions which yields a stronger mode of convergence-that of convergence in distribution-of the normalized sums of independent random variables to the counterpart $\delta_{2 n}$ of the "standard normal". Additionally, we no longer require that the random variables be identically distributed. Maintaining the style of our earlier work, we first state and prove the results for the case $n=2$, for which $p_{4}(t, x)$ is the fundamental solution of a fourth-order partial differential equation, and then restate the theorem more generally for arbitrary integers $n>2$.

2. Central limit theorem for the case $n=2$. We first consider the "signed probability density function" $p_{4}(1, x)$ corresponding to the signed distribution function $P_{4}$. The following results will be necessary for the derivation of our theorem.

Proposition 2.1. $p_{4}(1, x)$ satisfies the following:

(i) $p_{4}(1, x)$ belongs to the $S c h w a r z$ class $C_{\downarrow}^{\infty}$ of rapidly decreasing infinitely differentiable functions;

(ii) $p_{4}(1, x)$ decays asymptotically as $|x| \rightarrow \infty$ like $|x|^{-1 / 3} \exp \left\{-a|x|^{4 / 3}\right\}$. $\cos \left(b|x|^{4 / 3}\right)$, where $a=(3 / 8) 4^{-1 / 3}$ and $b=3^{1 / 2} a$.

Proof. Property (i) follows from the fact that $p_{4}(1, x)$ is the Fourier transform of a $C_{\downarrow}^{\infty}$ function. Property (ii) is derived in Hochberg [4, \$2] using the method of steepest descent.

By $C^{k}$ we will denote the set of functions $\left\{f: f, f^{\prime}, f^{\prime \prime}, \ldots, f^{(k)}\right.$ are bounded, continuous functions $\}$. For a random variable $x$ distributed by a positive or signed measure $P$, we let $E\{x\}=\int_{-\infty}^{\infty} x d P$ and $|E|\{x\}=\int_{-\infty}^{\infty} x|d P|$.

The main results of our paper now follow.

TheOREM 2.2 (CENTRAL LIMT THEOREM). Let $x_{i}, i=1,2, \ldots$, be independent random variables distributed by a signed measure $\mu$, such that

$$
\begin{aligned}
\int_{-\infty}^{\infty} d \mu & =1, \\
\int_{-\infty}^{\infty} x_{i}^{j} d \mu & =0, \quad j=1,2,3, \\
& =-4 !, \quad j=4, \\
\int_{-\infty}^{\infty}\left|x_{i}\right|^{5}|d \mu| & =\gamma_{i}<\infty, \\
\sum_{i=1}^{k} \gamma_{i} & =\Gamma_{k},
\end{aligned}
$$

and $\Gamma_{k} / k^{5 / 4} \rightarrow 0$. Let $S_{k}=x_{1}+x_{2}+\cdots+x_{k}$. Then, $S_{k} / k^{1 / 4}$ converges in distribution to the random variable $\delta_{4}$ whose distribution is given by $P_{4}$. 
Notation. $S_{k} / k^{1 / 4} \stackrel{d}{\rightarrow} \delta_{4}$ or $S_{k} / k^{1 / 4} \stackrel{d}{\rightarrow} P_{4}$ as $k \rightarrow \infty$. The latter is the "hybrid" notation of Billingsley [1, p. 24].

ProOf OF THE THEOREM. We adapt a technique suggested by Trotter's method of operators (Trotter [7]) for convergence of suitably normalized sums of random variables to the standard normal. Consider random functions $\left\{y_{i}, i>1\right\}$ distributed by the signed measure $P_{4}$ described above. Each $y_{i}$ has the same first four moments as do the $\left\{x_{i}, i>1\right\}$. Let all the $x_{i}$ and $y_{i}$ be independent, and let $z_{j}=y_{1}+\cdots+y_{j-1}+x_{j+1}+\cdots+x_{k}$ for $j=2, \ldots, k-1$, with $z_{1}=\sum_{i=2}^{k} x_{i}$ and $z_{k}=\sum_{i=1}^{k-1} y_{i}$. Clearly,

$$
\left[\varphi\left(\sum_{i=1}^{k} x_{i}\right)-\varphi\left(\sum_{i=1}^{k} y_{i}\right)\right]=\sum_{i=1}^{k}\left[\varphi\left(x_{i}+z_{i}\right)-\varphi\left(y_{i}+z_{i}\right)\right] .
$$

By Taylor's theorem for $\varphi \in C^{5}$,

$$
\begin{aligned}
\mid \varphi(x+z)-\left[\varphi(z)+\varphi^{\prime}(z) x\right. & \left.+\frac{1}{2} \varphi^{\prime \prime}(z) x^{2}+\frac{1}{6} \varphi^{\prime \prime \prime}(z) x^{3}+\frac{1}{24} \varphi^{\prime \prime \prime \prime}(z) x^{4}\right] \mid \\
& <\frac{1}{5 !}\left\|\varphi^{(5)}\right\|\left[|x|^{5}\right],
\end{aligned}
$$

where $\|f\|=\sup _{x \in R}|f(x)|$, from which we get, taking expectations,

$$
\begin{gathered}
\mid E\{\varphi(x+z)\}-E\{\varphi(z)\}-E\left\{\varphi^{\prime}(z)\right\} E\{x\}-\frac{1}{2} E\left\{\varphi^{\prime \prime}(z)\right\} E\left\{x^{2}\right\} \\
-\cdots-\frac{1}{24} E\left\{\varphi^{\prime \prime \prime \prime}(z)\right\} E\left\{x^{4}\right\} \mid<\frac{1}{5 !}\left\|\varphi^{(5)}\right\|\left[|E|\left\{|x|^{5}\right\}\right] .
\end{gathered}
$$

Replacing $x$ with $y$ and then subtracting yields

$$
|E\{\varphi(x+z)\}-E\{\varphi(y+z)\}|<\frac{1}{5 !}\left\|\varphi^{(5)}\right\|\left[|E|\left\{|x|^{5}\right\}+|E|\left\{|y|^{5}\right\}\right] .
$$

We now apply this result with $x=x_{i} / k^{1 / 4}, y=y_{i} / k^{1 / 4}, z=z_{i} / k^{1 / 4}$, and sum both sides of the inequality from $i=1$ to $k$. This gives

$$
\left|E\left\{\varphi\left(\frac{S_{k}}{k^{1 / 4}}\right)\right\}-E\left\{\varphi\left(\frac{\sum_{i-1}^{k} y_{i}}{k^{1 / 4}}\right)\right\}\right|<\frac{1}{5 !}\left\|\varphi^{(5)}\right\| \sum_{i=1}^{k}\left[\frac{\gamma_{i}}{k^{5 / 4}}+\frac{1}{k^{5 / 4}} \int_{-\infty}^{\infty}|x|^{5}\left|d P_{4}\right|\right] \text {. }
$$

But from Proposition 2.1 it follows that $|E|\left\{|x|^{5}\right\}=\int_{-\infty}^{\infty}|x|^{5}\left|d P_{4}\right|<\infty$, so the bound in (12) is at most $O\left(\Gamma_{k} / k^{5 / 4}\right)$, which approaches zero as $k$ becomes infinite by hypothesis. Thus for any $\varphi \in C^{5}$, we have

$$
\begin{aligned}
\lim _{k \rightarrow \infty} E\left\{\varphi\left(\frac{S_{k}}{k^{1 / 4}}\right)\right\} & =E\left\{\varphi\left(\frac{\sum_{i=1}^{k} y_{i}}{k^{1 / 4}}\right)\right\} \\
& =E\left\{\varphi\left(y_{j}\right)\right\}=\int_{-\infty}^{\infty} \varphi(x) p_{4}(1, x) d x
\end{aligned}
$$

Convergence in distribution of $S_{k} / k^{1 / 4}$ to $\delta_{4}$ now follows immediately from the general criteria for convergence; see, e.g., Billingsley [1] and Chung [2].

3. Central limit theorem for arbitrary values of $n$. We now restate the results of the previous section for random variables distributed by $P_{2 n}$, where $n$ is any 
arbitrary integer bigger than 1 . The proofs of these results are, of course, similar to those already presented.

Proposition 3.1. $p_{2 n}(1, x)$ satisfies the following:

(i) $p_{2 n}(1, x) \in C_{\downarrow}^{\infty}$;

(ii) $p_{2 n}(1, x) \sim|x|^{(1-n) /(2 n-1)} \exp \left\{-a_{n}|x|^{2 n /(2 n-1)}\right\} \cos \left(b_{n}|x|^{2 n /(2 n-1)}\right)$ as $|x| \rightarrow \infty$, where $a_{n}$ and $b_{n}$ are fixed constants depending on $n$.

Theorem 3.2 (Central Limt theorem). Let $x_{i}, i=1,2, \ldots$, be independent random variables distributed by a signed measure $\mu$, such that

$$
\begin{aligned}
\int_{-\infty}^{\infty} d \mu & =1, \\
\int_{-\infty}^{\infty} x_{i}^{j} d \mu & =0, \quad j=1,2, \ldots, 2 n-1, \\
& =(-1)^{n+1}(2 n) !, \quad j=2 n, \\
\int_{-\infty}^{\infty}\left|x_{i}\right|^{2 n+1}|d \mu| & =\gamma_{i}<\infty, \\
\sum_{i=1}^{k} \gamma_{i} & =\Gamma_{k},
\end{aligned}
$$

and $\Gamma_{k} / k^{(2 n+1) / 2 n} \rightarrow 0$. Let $S_{k}=x_{1}+x_{2}+\cdots+x_{k}$. Then $S_{k} / k^{1 / 2 n} \stackrel{d}{\rightarrow} \delta_{2 n}$ (or, equivalently, $P_{2 n}$ ) as $k \rightarrow \infty$.

\section{REFERENCES}

1. P. Billingsley, Convergence of probability measures, Wiley, New York, 1968.

2. K. L. Chung, A course in probability theory, Academic Press, New York, 1974.

3. R. Hersh, A class of "central limit theorems" for convolution products of generalized functions, Trans. Amer. Math. Soc. 140 (1969), 71-85.

4. K. J. Hochberg, A signed measure on path space related to Wiener measure, Ann. Probability 6 (1978), 433-458.

5. R. P. Kert, Random evolutions with underlying semi-Markov processes, Publ. Res. Inst. Math. Sci. Kyoto Univ. 14 (1978), 589-614.

6. Yu. P. Studnev, Some generalizations of limit theorems in probability theory, Theor. Probability Appl. (Teor. Verojatnost. i Primenen) 12 (1967), 668-672.

7. H. F. Trotter, An elementary proof of the central limit theorem, Arch. Math. (Basel) 10 (1959), 226-234.

8. A. I. Zukov, A limit theorem for difference operators, Uspehi Mat. Nauk 14 (1959), 129-136.

Department of Mathematics and Statistics, Case Western Resbrve University, Cleveland, OHIO 44106 